\title{
Perceptions of facilitators and barriers to mental health treatment engagement among decision- making competent adolescents in Greece
}

\section{E. Tsamadou}

Aristoteleio Panepistimio Thessalonikis: Aristoteleio Panepistemio Thessalonikes

\section{Polychronis Voultsos ( $\nabla$ pvoultsos@auth.gr)}

Aristotle University of Thessaloniki Department of Medicine: Aristoteleio Panepistemio Thessalonikes Tmema latrikes

\section{A. Emmanouilidis}

Aristotle University of Thessaloniki: Aristoteleio Panepistemio Thessalonikes

G. Ampatzoglou

Aristoteleio Panepistimio Thessalonikis: Aristoteleio Panepistemio Thessalonikes

Research article

Keywords: Adolescent psychotherapy engagement, shared decision making, decision making competent adolescents, (psycho-) therapist

Posted Date: February 23rd, 2021

DOI: https://doi.org/10.21203/rs.3.rs-220519/v1

License: (c) (1) This work is licensed under a Creative Commons Attribution 4.0 International License. Read Full License 


\section{Abstract \\ Background}

A subset of adolescents with mental disorders are likely to be (share) decision making competent and hence can make their treatment engagement more effective. Treatment engagement is decisive in achieving successful outcomes. However, there are high rates of drop-out in mental health settings.

\section{Aim}

This study aims at identifying barriers to or facilitators of mental health care engagement among adolescents with decision-making competence in Greece.

\section{Methods}

Participants were adolescents in therapy for mental disorders recruited from an outpatient tertiary hospital setting. First, potential participants with decision making competence were identified. Second, 50 participants were interviewed about their perceptions of treatment. The interviews were auto-recorded. A transcription verbatim and data analysis were conducted. All the requirements of a qualitative research and ethical considerations were observed.

\section{Results}

All the participants highlighted their experience with facilitators rather than barriers to their treatment engagement. Facilitators included: a) positive treatment outcomes and adolescent's perceived usefulness of treatment, b) a meaningful adolescent-therapist relationship, c) family's supportive role, d) symptoms and negative self-image, and e) developing social relationships and acceptance by peers. The participants equally highlighted the importance of getting rid of their symptoms and improve their socialization skills. Other facilitators included: achieving professional and personal goals in life, enhancing their independence and self-esteem / self-image, or even just confessing to another trustworthy person. Friends were reported as having a neutral or mild supportive role. The barriers included ineffective and unhelpful treatment, negative experiences with their therapist, relief from symptoms and mental health therapy-related stigma.

\section{Conclusion}

For the most part, the findings enhance prior studies. Participants highlighted facilitators rather than barriers to treatment engagement. For the most part, the findings enhance prior studies. However, we identified some nuances which might be used by therapists to enhance the adolescents' treatment 
engagement. For instance, bearing in their mind that by the subsiding of symptoms adolescents are most likely to terminate the treatment prematurely and highlighting the achievement of adolescents' future goals as well as the potential family's enhanced well-being because of the improvement of adolescents' mental health may contribute to reducing the attrition rate.

\section{Background}

Informed consent plays a crucial role in modern clinical practice. It constitutes a cornerstone of 'good medical practice'. A physician who fails to obtain valid informed consent before providing treatment can incur legal liability for committing a battery on the patient (with the exception of emergency treatment, which is always valid). By obtaining informed consent before providing medical treatment, the patient is being treated as autonomous moral agent. Informed consent requirement derives primarily from the principle of respect for autonomy.

Note, however, that mentally ill persons do no way lack decision-making competence for the only reason they are mentally disordered [1]. Mental disorder does not necessarily involve a loss of personal autonomy. It is important to bear in mind that perfect cognitive functions are not necessarily requirements for decision making capacity (DMC) [2]. Nevertheless, (perfect) cognitive functions are not always sufficient for having full DMC. Modern theory places emphasis on values and emotions rather than on cognitive functions when considering one's DMC. Values, preferences and emotions play an important role in decision-making process [3]. Importantly, adolescents with mental disorders are likely to be decision making competent.

Adolescence is a culturally defined concept without clear-cut starting (and ending) point [4]. While adolescents should be involved in treatment decisions to the extent possible $[5,6]$. Their decision-making competence should be assessed on an individual case. Below, we go into this topic.

Studies have demonstrated that a subset of adolescents aged 14 and older have the capacity to consent to medical treatments in specific contexts [7,8]. Legally-binding international texts such as the United Nations Convention on Rights of the Child (articles 12 and 13) and the Convention on Human Rights and Biomedicine (article 6) provide that the voices of children and adolescents should be heard and given due weight. Indeed, the rights of adolescents to be involved in treatment decisions have been expanded in recent years [9]. Adolescents' involvement in medical decisions is important to them DMI helps him or her become a better decision-maker in the future [6] and learn that he is a being of 'moral worth' [10].

As regards adult patients, it is extremely difficult for adults to define a cut-off point of consent for medical treatment [11]. That is particularly so when it comes to adolescents. There is no universal agreement on adolescents' decision-making competence (DMC). There is no chronological age of consent for medical treatment [12-14]. The existing consent frameworks do not specify a minimum age for which an adolescent might be considered competent to consent to medical treatment [15-16]. Importantly, it seems impossible to define a cut-off point of consent for medical treatment based on neuroscience [17]. There is variance in adolescents' decision-making competence. 
Adolescences' decision making competence is determined by a variety of factors related to adolescents themselves (i.e. developmental stage, literacy, culture, experience, health state), their context (i.e. parents that may be facilitators or barriers to adolescents' decision-making competence, peers, health professionals and the relationships between the adolescent and these individuals), and other situational factors [17-19]. Importantly, there is uniqueness and diversity in adolescence. Asymmetry in development of various structures in adolescent's brain is the main factor responsible for making adolescence a unique developmental period requiring a tailored response. Adolescents' decision-making capacity is determined by a variety of factors that interact with each other. Besides, it is context-dependent and changes over time ${ }^{5,19}$. Furthermore, other authors highlight the role of factors such as the development of skills and mature critical thinking, as well as the development of values, emotions and moral authority, their literacy, their culture, their previous experiences, the involvement of the family and the family relationships [5, 20-25]. Lastly, as briefly mentioned above, adolescent's decision-making competence fluctuates. It is arguably stated that 'decision-making, even of mature adolescents, may occasionally be flawed' [26]. Furthermore, there are considerable individual differences in rates of developmental maturation among adolescents [27]. Moreover, adolescents are profoundly influenced by other persons (especially parents, health professionals and peers) [28].

As adolescents' and children's medical decision making process is much more complex than adults' medical decision making process, it is particularly so when it comes to children and adolescents with mental disorders. Little is known about the capacity of adolescents with psychiatric mental disorders to consent to treatment [9]. Laufer (1992) [29] states that mental disorder in adolescence is difficult to be defined and opinions differ. It is estimated that approximately $20 \%$ of youth living in the United States (U.S.) have an emotional or behavioral disorder [30]. Moreover, it is noteworthy that there are high rates of drop-out in mental health settings [31]. 'Youth are particularly difficult to engage' [32]. Warnick et al. (2014) state that 'attrition in youth outpatient mental health clinics ranges from 30 to $70 \%$ and often occurs early in treatment' [33]. Voluntary treatment engagement of adolescents with mental disorders is crucial to establishing interactive and effective adolescent-therapist relationship and achieving successful therapeutic outcomes. SDM contributes to creating an optimum collaborative working involvement between therapist and adolescent. At any rate however, it is crucial to bear in mind that the politics which have been designed for what is called 'good medical practice' might not fit well with mental healthcare context [34].

To our knowledge, little research has been conducted up to now on factors that may be barriers to or facilitators of mental health care engagement among adolescents. In the absence of adequate specific empirical knowledge about these topics in Greece, this study aims at identifying and exploring factors that may be barriers to or facilitators of mental health care engagement among adolescents with decision-making competence in Greece. This study along with another (entitled: Epidemiological profile of decision making competent adolescents engaged in mental health therapy) were parts of a broader research project exploring the topic of consensual mental health treatment engagement among adolescents. 


\section{Research questions}

The grand tour question that delineates the focus of this study was the following:

What are the factors that may be perceived barriers to or facilitators of mental health care engagement among adolescents in Greece?

The secondary research questions were the following:

a) What are the major factors that can profoundly shape the attitude of adolescents with mental disorders towards their treatment?

b) What are the factors that can determine the consent of adolescents with mental disorders to their treatment?

c) What are the factors that can determine the treatment decision making of adolescents with mental disorders?

\section{Study Design}

The present work has been a prospective qualitative research study based on in-depth interviews with adolescent patients (outpatients) with psychiatric disorders. This qualitative descriptive study was conducted from June 2016 to December 2019 using conventional content analysis approach. Moreover, a psychometric evaluation of the adolescent patents who were willing to participate in our study was conducted. While the primary goal of psychometric assessment was the application of the exclusion criteria to our sample, we thought that a further statistical analysis of the psychometric assessment results might help us get better overall study results. In addition to the application of the exclusive criteria psychometric assessment was expected to help us identify more factors potentially affecting the patients' attitude towards their treatment and carry out a more nuanced and reliable qualitative analysis, namely, get better results from our qualitative data analysis. In this perspective, prior to participating in qualitative research, these patients were administered two psychometric scales: A) An intelligence test (Wechsler Intelligence Scale for Children, WISC III) [35] and a self-report measure of depression (Beck Depression Inventory, BDIII) [36]. The Greek version of both psychometric tests were considered reliable since these questionnaires have been validated in the Greek context $[37,38]$. Quantitative data presented below are from that survey.

\section{Sampling and data collection}

A total of 50 adolescents (aged 13-18 years, mean=14,8, SDs=1,616) with psychiatric disorders (outpatients attending their scheduled clinic appointments in Child and Adolescents Psychiatry department, tertial referral hospital Hippokratio of Thessaloniki) participated in the study. A broad range of mental disorders were included such as depression, obsessive-compulsive disorder, dysthymia, behavioral disorders, anorexia nervosa, panic disorder, bipolar disorder, autism spectrum disorder. All of 
the participants had been attending psychotherapy for at least four months, while most of them had been in psychotherapy for more than six months. No one of them was involuntarily referred by parents and other caregivers.

In the present study, we focused on adolescent patients' perceptions and attitudes as a determinant of the propensity to consent to and comply with their treatment. Purposive sampling was used according to the eligibility criteria set out below.

After having undergone the psychometric evaluation the members of our sample underwent in-depth interviews. We conducted in person interviews based on a semi-structured interview guide. Qualitative interviewing data were collected from 50 participants.

The inclusion criteria for participation in the study were: (1) being an outpatient with mental disorders, (2) being child or adolescent not older than 18 years, (3) being already engaged in a therapeutic relationship in our healthcare setting, and (4) being not involuntarily engaged in this therapeutic relationship. The exclusion criteria aimed at creating a pool of participants who were consensually engaged in treatment and were able to undertake the task of being interviewee in an effective interviewing. We initially excluded patients with severe depression, psychosis, moderate and severe mental disorder, severe autism spectrum disorder without sufficient language development. The exclusion criteria for participation in the study were: (1) incapability of using the Greek language, (2) having very low literacy level (determined empirically), namely, a literacy level below what is needed for effective interviewing. Adolescents decision making competence was assessed on an individual case. Therefore, exclusion criteria were the following: (3) lack of an adequately developed set of values (explored through interviews), (4) very low intelligence or (5) severe depression symptoms that may have negative impact on cognitive functions and decisionmaking process as well. Therefore, patients with severe disorders of cognitive functions or severe emotional (major depressive) disorders were excluded. Not only these disorders may have a profoundly negative impact on participants' DMC, but also these disorders may reduce the reliability and effectiveness of interviewing. In this perspective, we determined a threshold value (cutoff points) to identify those patients with 'very low intelligence' or 'severe depression symptoms', namely, those who were eligible to become participants in the qualitative research. The cutoff point used for the WISC III was $<54$ and the cutoff point used for the BDI II was $>31$. If the total score was less than these threshold values, the intelligence and depression symptoms were classified as 'very low' or 'severe', respectively. Potential participants were administered two psychometric scales: A) An intelligence test (Wechsler Intelligence Scale for Children, WISC III) and a self-report measure of depression (Beck Depression Inventory, BDIII). The Greek version of both psychometric tests were considered reliable since these questionnaires have been validated in the Greek context $(37,38)$.

Then, in a second step, adolescents whose intelligence and depression symptoms were not classified as 'very low' or 'severe' respectively, were clinically assessed whether they had developed a set of (not pathogenic) values strictly and stably over time allied to their narrative identity, as well as whether they had adequate (though not extreme) emotions. The assessment was not difficult provided that the 
adolescents had already been in therapy for many months (at least four months, mostly six months). Moreover, we found out whether they had developed practical wisdom (focusing on 'knowing the right thing to do in the concrete situation'). (Widdershoven et al., 2017)(39). More precisely, we clinically assessed whether they were able to find a balance between various values and extreme emotions, and 'enact them in personal life' (39). While the participants in our study did not lack the capacity of cognitive reasoning and had adequate values and emotions, we considered that having practical insight and being able 'to handle the situation and live a meaningful life' might further confirm their decision making competence. We did so because adolescents with mental disorders are in an extremely blurry zone between competence and incompetence. We used the ICD-10 to obtain diagnosis for mental health disorders.

Note, however, that this study along with another (entitled: Self-perceived barriers to and facilitators of voluntary health care treatment engagement among adolescent outpatients) were parts of a broader research project exploring the topic of mental health treatment engagement among decision making competent adolescents. For this reason, in both studies, we assessed the adolescents' decision making competence in the same way using exactly the same steps.

Thus, participants who differed in age, gender, educational background, experience and type of mental disorder were recruited continuously during the project by the main researcher (ET).

The researchers managed to achieve a cluster random sample. To gain a deep and comprehensive understanding of underlying phenomena that drive psychiatric adolescents' attitudes towards their own treatment, we strived to achieve an overall sample consisting of participants coming from a wide range of mental health conditions (corresponding to a wide range of types of mental disorder), weighed against a practical wish to recruit the greatest possible number of participants from each type of mental disorder.

A total of 52 patients were initially conducted by the main researcher (first author in this paper). While 50 patients agreed to be interviewed, 2 patients chose not to participate in the research study. No one patient initially agreed to participate and then changed their mind and declined participation.

\section{Interviews}

The interview guide reflected the overarching aim of the study: to investigate any barriers to and facilitators of adolescents' capacity to decide and give their valid consent to their treatment. The interviews were semi-structured and started by guide questions like, What were your perceptions about what is good or bad with your treatment?; Why did you not give consent to receive the treatment offered to you? ?; What are your views about your treatment? How would it matter to you if you did not receive the treatment offered to you? Have you ever felt concerned about your treatment? How would it matter to you if you had to receive a drug? What do you believe are the main reasons for which you have to comply with the treatment offered? Please describe to me any significant experience you have had dealing with your problems. Have you ever had an experience with similar treatments for example, a personal experience or with family/friends? How did you decide whether you should undergo treatment? How did 
this experience affect your attitude towards your treatment? What is or should be the role of your therapist and the relationship between you and your therapist? What would that relationship look like? How do you feel about this? What do you expect from your therapist in relation to your treatment? How did you find the behavior of your therapist in caring for you? What was your relatives' / peers' role in your decision? What kind of reactions to your family / peers have you observed in yourself? and so on. The interview guide questions were developed specifically for this study (tailored to the participants' conditions), with the possibility of qualifying probing questions which were not listed in the interview guide, depending on the course of the interview. The topics set out in the interview guide included reasons, feelings, views, experiences and understanding. The interviews were focused, among others, on elucidating the reasons why patients decided to undergo medical treatment, their comprehension relating to treatment design and processes, and their experiences of taking part in the therapeutic alliance with their therapist and receiving a drug. Moreover, the interviews included questions about the participants' experiences and perceptions of treatment, as well as questions about their view on social and family relationships, future targets and goals. Study participants were also asked about difficulties in complying with the treatment offered.

As data analysis commenced while the trial was ongoing, new topics were highlighted during the initial few interviews and emerged from data analysis. In subsequent interviews these topics became the subject of additional questions (added to the interview guide) to further confirm the trustworthiness of our research data.

Participants were encouraged to expand upon issues they considered most relevant and speak as freely as possible about them. Participants were encouraged to elaborate on their initial responses to the questions. The interviewer made every effort not to interrupt the interviewer while speaking, or to not disturb the interviewer while remaining silent. She tried her utmost not to ask leading questions.

All interviews were carried out in person by the first author (ET), a specialist in child and adolescent psychiatry possessing a Master of Medical Ethics and experienced in qualitative interviewing. Data were collected through in-depth individual semi-structured interviews. Relevant field notes were written before and after interviews by the interviewer to help produce a comprehensive set of insightful findings.

Patients were given the choice of being interviewed either at their home or at their therapist's general practice. Interviews were held at interviewees' preferred time. During the interview only the interviewer and the interviewee were present, with the exception of few patients who were accompanied in the interview by their parents, who for the most part stayed silent apart from their brief input. This input was not analysed and used for the paper.

The interview language was Greek and interviews were conducted between June 2016 and December 2019. Interviews lasted approximately between 30 and $45 \mathrm{~min}$. The mean length of the interviews was 35 min. Interviews were audio recorded and transcribed verbatim by the first author into written Greek. 
Although the researcher had a clinical background, she assumed the researcher role for the interviews. She did not hold any strong views about medical ethics and remained neutral on issues that were discussed with the patient. She contacted the patients and spent time beforehand to gain participants' trust and ensure trustworthiness in the study. She answered any questions that the patients asked. Reflexive thinking was applied throughout the research process to reduce unintended personal bias. She took great effort to make interviews feel more like a conversation and less like an interrogation (especially from the adolescent patients' perspective).

The number of participants was not set from the beginning. Data collection ceased only when data saturation was reached, a point where no additional information was obtained from further interviews. Data collection continued until after the forty-five interviews. Five more interviews were also conducted to ensure the data saturation.

\section{Data analysis}

The analysis was mainly performed by the first author. The co-authors contributed to the analysis from their respective point as bioethicist, clinician, or psychologist. Moreover, each one of us engaged with other researchers to limit research bias.

As our data did not involve particularly large volumes of text and we placed great weigh on the interpretation of the 'latent content' of texts and on the subjective understanding of "patterns, themes, and categories important to asocial reality" [40], the interviews were analysed with conventional qualitative content analysis as suggested by Graneheim and Lundman (2003) [41]. With respect to the aim of the study, the analysis was focused on identifying perceptions of the adolescent patients towards their treatment. In this sense the analysis was purposive.

We took great care to ensure the validity and reliability of the study according to Gibbs (2007) [42]. In addition, we used strategies to minimize reflexivity throughout the project. For instance, throughout the content data analysis, we drew upon our own clinical experiences in a reflexive manner. Furthermore, credibility was established using prolonged engagement, and maximum variance of participants' selection. Moreover, transferability was achieved via the provision of a rich description of data collection, analysis processes and findings to allow the readers to match the findings with their contexts. Trustworthiness of a qualitative research study involves establishing credibility and transferability (as well as dependability, confirmability and authenticity) $[43,44]$.

As the purpose of our study was not early theory testing, we used inductive content analysis, namely, an approach moving from the specific to the general [45].

After each interview was transcribed word by word, in the first step, each interview transcript was read through carefully and repeatedly to obtain a good overall sense of the whole transcript and impression of its content [46]. In the second step, units expressing a meaning were identified in each interview transcript, and similar in meaning units were coded. The researchers constantly compared data to ensure 
the codes were used consistently [47]. In the third step, codes similar in meaning were grouped into subcategories. In the fourth step, subcategories compared with each other and the latent data content were condensed into broader categories. The final categories were refined by all authors through ensuring a clear difference between categories and subcategories. The lists of categories were grouped under higher order headings. The categories were grouped into twelve prevailing themes as final product of the analysis: 1) Five major facilitators: a) positive treatment outcomes and adolescent's perceived usefulness of treatment, b) a meaningful adolescent-therapist relationship, c) family's supportive role, d) symptoms and negative self-image, and e) developing social relationships and acceptance by peers. 2) Three minor facilitators: a) achieving professional and personal goals in life, b) enhancing their independence and self-esteem / self-image, and c) just confessing to another trustworthy person.3) Four barriers: ineffective and unhelpful treatment, negative experiences with their therapist, relief from symptoms and mental health therapy-related stigma.

Disagreements between the authors that arose during the data analysis were easily addressed with reexamination of the data and further discussion.

Data analysis carried out using NVivo qualitative data analysis software version 9 released in 2010 .

\section{Ethical considerations}

The authors obtained adolescent consent and parental consent for adolescent participants. If adolescents and their parents were willing to participate they were given adequate information about the design, purpose, nature and confidentiality of the study, including that participation was voluntary and that consent could be withdrawn at any time during the course of the study. Verbal informed consent to participate was then obtained from each participant and his or her parent(s) prior to participating in this study and documented in recording at the time of the interviews. Anonymity and confidentiality have been maintained throughout the study. Interview data were anonymized during transcription.

In order to preserve their anonymity, no names are used in this paper. The interviews were registered and stored in a strictly confidential fashion. The study and consent procedure were approved by Ethics Committee affiliated to Aristotle University of Thessaloniki, Faculty of Health Sciences, School of Medicine (No: 9.302 / 12-07-2017).

\section{Results}

All of the participants in interviews highlighted their experience with facilitators of rather than barriers to their treatment engagement. Among the most highlighted facilitators identified were included: a) positive treatment outcomes and perceived usefulness and effectiveness of the treatment, b) a 'good' adolescenttherapist interaction, namely, a meaningful, close, trustful, warm, open, communicative and familiar relationship with a therapist in which they feel comfortable, c) family's supportive or active role in favor of adolescents' treatment engagement, d) getting rid of their symptoms and negative self-image, and e) developing social relationships and improving their social interactions and acceptance by peers. The 
participants equally highlighted the importance of getting rid of their symptoms and improve their socialization skills. Other facilitators were: achieving professional and personal goals in life (although the vast majority of participants disconnected these goals from therapy), enhancing their independence and self-esteem / self-image, or even just confessing to another trustworthy person. Friends were reported as having a role ranging from neutral to mildly supportive.

Among the barriers to treatment engagement participants described the relief from symptoms that can cause a high attrition rate, 'bad' relationship with the therapist, negative experiences with mental health treatment perceived as ineffective and unhelpful, and fear of mental health-related stigma.

Below are presented the themes and sub-themes that emerged from our interview data analysis [For additional representative quotes see Additional File 1].

\section{Recognition of the treatment and willingness to undergo it (and underlying rationales)}

In the sample of adolescents examined, almost all participants considered treatment in a positive view. The vast majority of the minors stand undoubtedly in favor of therapy, as they search for help to handle their difficulties and get free of symptoms that potentially limit and change their daily functionality, their plans in personal and professional fields and obstruct social relations development.

The vast majority of participants not only was committed to therapy, but also invested in it to a varying degree. One participant, while she recognized the beneficial role of therapy and declared her commitment to it, stressed that she would avoid investing in therapy.

I don't think of anything special, it's a new experience as well, which will help me; it will get somewhere, but I don't need to have a special feeling for it... are you scared of something? fast answer: no (participant 8, girl 15 years old, F50)

Importantly, while the vast majority of participants clearly acknowledge the benefit they receive and desire treatment, many participants were minded to terminate therapy prematurely, if symptoms recede. One participant expressed the desire to conclude treatment quickly. Perceived ineffective treatment may be a good reason for terminating therapy prematurely. Interestingly, the adolescents were ready to determine the terms and conditions of their therapeutic relationship.

\section{The fear of mental health-related stigma as a barrier}

The need for confidentiality in the adolescent-therapist relationship, expressed by many participants (see below), underlies the fear of social stigmatization. The same applies concerning the need for confidentiality between adolescents and their relatives (see below).

For example, one participant while talking to the interviewer reflected: 
I don't have a problem because this won't be known and it will also help me...yeah, since you are not going to say it to anyone, I don't need to worry... (Participant 3, girl 14yo, F39)

\section{The fear of mental disorder as a facilitator}

The need of adolescents to believe that they are not dealing with a serious issue seems to function as a relief and encouragement to treatment. For example, two participants reflected:

"Since I know the reason I'm going and I am aware of my condition, that it's not a serious problem or something to worry about, I don't see it negatively, as another child might, and I am OK with it and myself and I don't worry" (Participant 13, boy 16yo, F40, F51)

"I don't have a special problem, but I think it would be good to discuss my problems with someone" (Participant 26, boy 13,5yo, F39)

For this reason they may transfer the initiative of seeking treatment exclusively to parents (see below, role of family)

\section{Seeking independence}

Two participants stated:

"[...] I expect some things I want to be changed will change (hesitates)... when I go to High School, to be able to decide for myself and not follow my therapist's instructions"(Participant 49, boy 13yo, F42)

"[...] I think that... I do this... to be gradually better till I become independent and can find my path alone... I fear of regressing... I think it will help me be more independent regarding the things that scare me and become able to confront them alone... I'd like to develop my sociality and my knowledge mainly" (Participant 4, girl 17yo, F50)

\section{Need to enhance their self-esteem and improve their self- image}

Some adolescents were motivated to treatment out oftheir need to increase their self-esteem and improve their perception of themselves or their lives.

The following comments illustrate this point:

"[...] it makes me think more rationally and I deal better with the issue I'm having... as I will be better with myself, I will also be better with others" (Participant 13, boy 16yo, F40, F51)

"[...] I become better person and I believe I'll make the right choices about my life... I'll have a better perception of things..." (Participant 9, boy 17yo, F39)

\section{Treatment as a presupposition for the realization of future plans}


For 3 participants, treatment was considered a presupposition for the realization of personal and professional future plans.

Typical comments included:

"... I think that if I hadn't selected to be treated, some future plans would not have been accomplished" (Participant 9, boy 17yo, F39)

"My plans for the future were mainly the incentive to accept the proposed treatment" (Participant 4, girl 17yo, F50)

"I believe that [the treatment] will help me achieve some goals in my life" (Participant 12, boy 15yo, socialization problems)

However, the vast majority of participants did not connect the achievement of future goals with the present treatment, despite the gravity of their current condition. The initial feeling of "invincible" and "temporary" dominates the adolescent psyche and may help them deal with weaknesses.

The majority of adolescents set studies and future career preferences as main priority. Leaving the family home, moving abroad for studies and creating their own family express the deeper need for independence and autonomy. A typical comment was:

"... study here and leave for Germany, study there as well" (Participant 4, girl 17yo, F50)

\section{Treatment as confession}

For example, three participants reflected:

“... I don't exactly know [the problem], but speaking helps get it out of you" (Participant 3, girl 14yo, F39)

"... I expect to describe what I've been through from my father... to say what I have inside of me... [and to be told] how I should behave" (Participant 41, girl 15yo, F39)

"I think it's something good, cause there were periods previously that I was much worse, it's something that helped me, I believe it's good... I'm being helped initially because I express myself to someone, I say what I feel... those things I'm afraid to say to my friends and my parents" (Participant 47, girl 16yo, F32) Relief from symptoms - negative consequences of the disorder

Participants were classified in the following categories of mental disorders:

$44,9 \%$ in the F30-F39 category, [manic episode, bipolar affectional disorder, depressive episode, recurrent depressive disorder, persistent mood disorders (cyclothymia, dysthymia), other mood disorders, (ICD-10, 1993]. 
$20,4 \%$ in the F40-F48 category, [neurotic and somatoform disorders, such as phobic anxiety disorder, anxiety disorders, obsessive - compulsive disorder, adjustment disorders, dissociative disorders].

10,2\% in the F50-F59 category, [eating disorders, sleep disorders, sexual dysfunction, substance abuse. Importantly, this category includes anorexia nervosa].

2\% in the F60-F69 and F70-F79 categories, [personality disorders and mental retardation respectively].

$10,2 \%$ in the F80-F89 category, [developmental disorders of speech and language, disorders of scholastic skills and pervasive developmental disorders].

4,1\% in the F90-F99 category, [hyperkinetic disorders, conduct disorders, emotional disorders with onset specific to childhood, tic disorders, disorders of social functioning with onset specific to childhood].

$6,1 \%$ in the Double Diagnoses category

A total of $22.4 \%$ of the participants in our study had sleep disorders. Furthermore, $44,9 \%$ of the adolescents in our study were affected by mood disorders and increased rates of pessimistic symptoms $(36,7 \%)$, energy reduction $(30,6 \%)$, concentration difficulties $(28,6 \%)$ were observed. It is notable that although the participants in our study stressed the need for developing social relations and becoming accepted by peers, no-one (even those of higher age) mentioned the creation of erotic relations as a therapy goal. In a relative question during their evaluation, they were indifferent to such issues.

\section{a) Focusing on personal problems}

Some mentioned that they wanted to be better off in general and to effectively deal with the difficulties and challenges of their life.

The following were typical comments:

"I expect to become better and I'm not afraid of anything" (Participant 19, boy 16yo, F42)

"I believe it will quite help me... I expect it will relieve me and I'm not afraid of anything" (Participant 3, girl 14yo, F39)

"I believe it will help me... feel better" (Participant 43, girl 17yo, F32)

\section{b) Relief from symptoms and improvement of socialisation}

Typical comments included:

"[I want to] be able to develop friendships with other children... to start a conversation, to be myself, because many times I'm afraid to speak" (Participant 35, boy 17yo, F84.1)

"The suggested treatment could help me in various phobias, so as to get over them... if I have issues with my friends, treatment will help me deal with them... I'd like to develop friendships" (Participant 21, boy 
"[l want to] solve some problems I had with my classmates at school" (Participant 25, girl 13yo, F39)

\section{c) Acceptance from the social environment (especially peers)}

Success in the goal of being accepted by the social environment and especially peers is a main motive for accepting treatment.

\section{Adolescents' reasons for refusing (or being unwilling to continue) treatment}

We identified that adolescent patients might refuse or be unwilling to continue their treatment for a variety of distinct reasons

\section{a) Relief from symptoms as a barrier to therapy engagement: risk of early withdrawal from therapy}

The improvement of symptoms consists of another element for the interruption of the therapeutic relation, since whatever stigmatization from the mental illness is eliminated as well as the need for follow-up.

“...if I feel better, I'll stop [treatment]" (Participant 33, girl, 17yo, F42)

"... if I feel it [therapy] helps me more and I want to finish... I don't want it much, I want to finish...it helped me already, I don't want anything more... I'm afraid to stop... some classmates make fun of me" (Participant 10, boy 13yo, F84.5)

“...treatment interruption, when I see I don't need it any more" (Participant 3, girl, 14yo, F39)

"I believe I would interrupt treatment, if I was reaching a good, very good point, that I wouldn't need anything more or I had reached the point I wanted" (Participant 13, boy, 16yo, F40, F51)

\section{b) Perceived ineffectiveness of therapy as a barrier to therapy engagement}

The following comments illustrate this point:

"I'd interrupt it, if I saw it didn't help me" (Participant 12, boy 15yo, socialization problems)

"...[I'd interrupt it] if it is extremely difficult to follow it or I didn't notice any result in some time" (Participant 4, girl 17yo, F50) 
Some participants relate the perceived ineffectiveness of therapy to their therapist

"...the relationship with the therapist made me continue... if it didn't help me \{l'd stop treatment\}" (Participant 2, boy 13yo, F51.3)

"I have applied what she [the therapist] proposes to me and it leads to good results and they help me personally, so whatever she says, I listen" (Participant 8, girl 15yo, F50)

"...some kind of health problem it could cause me, what the therapist recommends" (Participant 24, boy 13yo, F90)

\section{c) Adolescents pose their own terms of a "good relationship" with the therapist, as a precondition for the continuing of treatment.}

Adolescents stop treatment, if it opposes their values, their personal beliefs and ideology, if it contradicts basic parts of their character. The need to preserve their selves, instead of a radical change through treatment, seems to be the key point for adolescents. They interrupt treatment unilaterally as an equal partner.

The following comments illustrate this point:

“something I can't accept or it doesn't fit my personality or character" (Participant 39, girl 15yo, F40 panic attacks)

"I stopped because I was getting frustrated when we disagreed on something" (Participant 25, girl 13yo, F39)

Moreover, adolescents desire clarity on what the therapist says.

"...if it is clear what she says, then yes" (Participant 5, girl 12,5yo, F45, F40)

And to feel well with him.

"It would make me interrupt it [treatment]... when the doctor would make me feel uncomfortable" (Participant 12, boy 15yo, socialization problems)

"My relationship with my therapist plays major role in my decision to accept the proposed treatment, because if the relationship is bad, then the treatment will be rather uncomfortable" (Participant 21, boy 17yo, F90)

"If I had a different doctor where we wouldn't have such communication and a good relationship, it'd be more difficult for me to apply some things" (Participant 47, girl 16yo, F32) 
It is noticeable that while adolescents seek a warm relationship with their therapist, bringing therapist's personal experiences in that relationship may have a tremendous negative impact on adolescent's treatment engagement. For example, one participant considered this a 'casus belli' and expressed a cynical view, alleging that

"if she starts bringing her personal stuff in the conversation, that's a red flag for me" (Participant 14, girl 14yo, F32)

\section{d) Some participants are strongly attached to treatment (invest in it) and find it difficult to see reasons to interrupt it}

Typical comments included

"...if, knock wood, I had something and was in hospital for days, if someone was injured, then I believe l'd interrupt..." (Participant 49, boy 13yo, F42)

"Nothing would make me stop treatment" (Participant 31, girl 16yo, F50)

Some participants strongly associate their steady commitment towards therapy with their good relationship with their therapist

"I don't think I could interrupt my treatment without my therapist saying so" (Participant 18, girl 18yo, bipolar)

"I don't think that something would change about the decision I make, regarding my relationship with her" (Participant 3, girl 14yo, F39)

\section{The crucial role of establishing adolescent-therapist relationship: A strong barrier or a strong facilitator}

The adolescent-care provider relationship appears to be very specific and essential for their treatment engagement and hence providing effective treatment. Establishing an adolescent-therapist relationship perceived as 'good' by adolescents may be a strong facilitator of adolescents' treatment engagement, whereas a perceived 'bad' relationship with the therapist constitutes a strong barrier to adolescents' treatment engagement.

The adolescent is initially cautious, curious, expecting that the therapist will persuade him about their collaboration and setting from the beginning the precondition of "comfort" with his therapist, so as to "succumb" to therapeutic guidance. Adolescents search for specific characteristics in the therapist. Adolescents request that therapists have a cold demeanor and do strive to create a trustful relationship with their adolescent patients. Trust towards the therapist and his confidentiality are important elements 
for the continuation of the therapeutic relationship and a prerequisite for the adolescent. Personal liking for the therapist is often an essential condition for continuing treatment. "If I didn't like her as much as I do, l'd be more hesitant to continue treatment". The need for confidentiality, so as to avoid social comments, is a characteristic of their relationship with the therapist. The adolescent needs to feel the specialist close to him, to feel confidence, comfort and intimacy, to speak "the same language", because that way he feels that he's not alone with the mental illness. However, the specialist is not an expert defining the life of the adolescent through a paternalistic model of doctor - patient relationship; they both decide in common. It is characteristic that two participants used the terms "collaborate [with the therapist]" (participant 33, girl 17yo, F42) and "[the therapist] is cooperative" (participant 20, girl 18yo, panic attacks).

\section{a) The (perceived) 'good' adolescent-therapist relationship and underlying rationales}

The vast majority of participants in our study did not describe any actual concerns about their therapist. Participants that perceived the relationship with their therapist as 'good' stated that their therapist was

"very good" and that they developed a friendly relationship of trust with him.

Typical comments included:

"I trust her" (Participant13, boy 16yo, F40, F51)

"Very good and friendly" (Participant 24, boy 13yo, F90)

"She's very good, I solve my problems" (Participant 10, boy 13yo, F84.5)

"Our relationship is good, I can trust her with many things and talk about my personal life and I consider her confidential" (Participant 4, girl 17yo, F50)

“... I love her much" (Participant 17, boy 14yo, F84.1)

Participants reported the following reasons as fundamental to establishing a good relationship with their therapist: "She understands me", "She listens to me" (that is she puts up with me), "I can speak freely", "She offers solutions", "I feel comfortable" are some of the phrases used by participants that perceive the relationship with their therapist as good.

Typical comments included:

"I feel intimate, she advises how to deal with my difficulties... I feel her close to me and I trust her" (Participant 45, girl 13yo, F34)

"My relationship with the therapist is very good, he makes me feel intimate with him" (Participant 12, boy $15 y o$, socialization problems) 
"My relationship with my therapist is very good... she is very cooperative... she speaks so nicely and clearly and I have understood that she'll probably help me with the treatment she offers me" (Participant 18, girl 18yo, bipolar)

One participant stated explicitly that establishment of a 'good' adolescent-therapist relationship as a facilitator of engagement in therapy

"I think that if the therapist succeeds in creating an atmosphere of trust and confidence, then it'd be easier for me to accept the proposed treatment" (Participant 4, girl 17yo, F50)

\section{b) Reservations towards the therapist}

Some mild reservations towards their therapist expressed by a few participants did not amount to actual concerns.

Four participants expressed a wait-and-see attitude towards their therapist. For example, the following comments are typical

"I have the judgment to understand if what she proposes to me will help me or not. For the time being I haven't noticed being affected. I see her positively" (Participant 13, boy 16yo, F40, F51)

Furthermore, some few participants said they had taken a weak liking to their therapist. Typical comments included:

"She was nice, nothing special" (Participant 25, boy 13yo, F39)

\section{The role of family ranges from only mildly supportive to strongly supportive}

All participants stated they had a good relationship with their family. Very few were slightly cautious with this statement. The role of the family in the treatment continues to be important, despite the tendency for independence which characterizes adolescence.

To a greater or lesser extent, the family helps adolescents to become willing to undergo treatment. While some adolescents assumed initiative for seeking therapy contact in some other cases the initiative for seeking therapy contact was assumed by family. However, none of the participants reported that they were feeling forced into psychotherapy. In almost all of the cases adolescents were backed by family. The adolescents showed a positive attitude towards the supportive role of family.

Many participants mentioned that family had a supportive role and amplified their therapeutic request.

Typical comments included: 
"They'll influence me positively because I believe they'll support me and they'd surely suggest it themselves" (Participant 12, boy 15yo, socialization issues)

Two participants clearly pointed out their own initiative in seeking treatment, thus minimizing the supportive role of the family.

"I had asked to come" (Participant 14, girl 14yo, F32)

"It was more my decision" (Participant 25, girl 13yo, F39)

In some cases, family had the principal initiative in seeking treatment and urging and persuading the adolescent.

"...no, my mom told me to come after she assured me that there's no problem and I also believe that there's no problem" (Participant 26, boy 13,5yo, F39)

"My relationship with my mom, that persuaded me" (Participant 10, boy 13yo, F84.5)

Some adolescents wanted to give joy to their family through the therapy.

"Since I understand what is good for me, it'll also be good for my parents cause they'll watch me get better" (Participant 13, boy 16yo, F40, F51)

"I believe that my decision will bond us more and I'll have the support of my parents for anything I want to do" (Participant 3, girl 14yo, F39)

One participant said that he expects treatment will improve his attitude towards his family and thus his relationship with them.

(reduces intrafamily conflicts??).

"My relationship with my family contributes to my decision to undergo the proposed treatment, because if something that happens in my family bothers me, treatment will help me to not be bothered any more." (Participant 21, boy 17yo, F90)

\section{The role of peers ranges from 'not-so-neutral' to mildly supportive}

The vast majority have friends and quite a few of them, however most participants chose not to announce to their friendly environment their problem and the fact they're in treatment. A lot of participants distinguish between their close trusted friends and the not so close ones. Close friends may know about the minor's therapeutic relationship and they potentially act supportively with emotions of solidarity, understanding and motivation. Most participants mentioned that their attitude towards 
treatment was not influenced by their friends. Some few participants felt support (participant 38, boy 17yo, panic attacks, stress; participant 8, girl 15yo, F50; participant 28, girl 15yo, personality disorder) or were urged towards treatment by friends (participant 14, girl 14yo, F32) or stated they pursued treatment to have a good relationship with their friends.

Typical comments included:

"If I have problems with my friends, treatment will help me deal with them" (Participant 21, boy 17yo, F90)

"... it's such a case, because the relationship I have with my friends encourages me to try not to be cut off by them again". Moreover, the participant clarified "the relations I have with my friends are generally good, but there are some friends with whom I can talk about personal issues, I trust them more and they encourage me" (Participant 4, girl 17yo, F50)

However, no participant stated that friends played a crucial role in seeking treatment and compliance with it.

\section{Discussion}

\section{Seeking treatment}

All participants had a positive attitude towards treatment. The vast majority of the participants in our research clearly acknowledge the benefit from receiving treatment and express explicit desire to undergo treatment. This is not in consistency with the findings of prior studies according to which adolescents frequently do not perceive the need for psycho-therapy $[27,48]$. It is argued that adolescents may lack cognitive abilities and experience to fully understand the rationale behind treatment and doubt that it will have any meaningful impact on them [27]. Furthermore, it is argued that adolescents are less able than adults to assimilate and integrate, to analyze, synthesize and evaluate the information provided, even though they may completely recognize the (short-term but not the long-term) benefits of the recommended treatment [9]. Moreover, it should be highlighted that Schachter, Kleinman and Harvey (2005) state that 'it is unclear how well adolescents with psychiatric problems appreciate their disorder and treatment recommendations and whether this situation is unique to adolescents or applies to adults as well' [49]. Note, however, that we included only adolescents that we considered competent to make decisions and were already in therapy. As anticipated above (in Introduction section), a subset of adolescents with mental disorders are likely to be decision making competent in specific contexts. These adolescents can be effectively involved in shared decision making and hence effectively engage in their treatment. To that effect, many theorists suggest that 'children may have far more potential to understand complex illness concepts than they have previously been given credit for' [50].

Quite some of the participants were of the opinion that the resolution of symptoms would be a good reason for attrition from (premature termination of) therapy, which in turn, nay hinder the effective delivery of mental health services [51]. It is important that quite a few adolescents seem to try to "have 
control" of the therapeutic relationship, setting their own terms of treatment delivery, which they mainly relate to the quality of their relationship with the therapist (see below). This corresponds with the fact that during adolescence there is desire for immediate results and adolescents focus on short-term outcomes but also with egocentrism of adolescence. The adolescents' perceived limits to their freedom to choose, perceived vulnerability to psychotherapy regarded as an effort to control them and conflict with their striving for autonomy, as well as the stereotype-based inaccurate impressions of psychotherapy, may be significant barriers to therapy engagement. It is particularly so when adolescents in therapy fail to perceive themselves as needing therapy and participate in therapy because others want them to be in therapy [27]. Adolescents have a propensity towards risk-taking and short-term reward (reward reactivity) due to asymmetry in development of various structures in adolescent's brain [13,17]. Besides, adolescence is characterized by the perception of invincibility and egocentrism (egocentric interference from self-perspective) [52,53]. A lot of participants clearly and undoubtedly state that they are afraid of nothing. Naturally, this agrees with the feeling of invincibility and risk taking during adolescence.

As all of the participants were actively engaged in therapy, it is most likely that their prevalent symptoms are potential facilitators of their engagement in therapy.

Lastly, it is noteworthy that our participants did not hold beliefs about mental health illness, mental health treatment or terrifying hospital experiences that contributed to poor treatment engagement (as Stafford and Draucker, 2020 found in their study) [48].

\section{Relationship with the therapist}

The vast majority of participants in our study reported that they had developed a meaningful connection with their therapist. They said that this was a significant facilitator of their therapy engagement. They stressed their belief that negative experiences with their therapists would be a major barrier to their therapy engagement. Our findings were in consistency with precious studies $[27,48]$. Bibliography has acknowledged long ago the adolescent-therapist relationship as crucial for the adolescent's engagement to treatment [27]. Adolescents' perceptions of therapists predict therapeutic outcomes [27]. Treatment engagement is decisive in forming an effective therapeutic process and 'may be particularly relevant early in treatment' [33]. The better the treatment engagement, the more favorable therapy outcomes may occur.

\section{Confidentiality}

Several participants mention trust in the therapist. Adolescents are more likely to seek health care if their provider assures confidentiality, but in providing confidential care a balance should be considered between the needs of the adolescent patient, parents, and provider [54]. In literature, distrust towards psycho-therapist is most commonly reported as barrier to therapy engagement in Latino adult populations $[48,55-62]$.

\section{Establishing reciprocal interaction and warm adolescent-therapist relationship}


Participants in our study are in need of a humane, approachable therapist who will collaborate with them treating them as equals. Therapeutic engagement is 'a reciprocal interaction in which both therapist and client(s) have a responsibility for establishing an effective rapport', namely, creating an optimum collaborative working involvement between therapist and adolescent [27]. Adolescents should be offered a way of building rapport with the therapist. They should participate fully and engage in positive interactions to achieve a successful therapy. Healthcare provider ability to engage adolescents, increases the possibilities for continuation of treatment [63]. Positive attitudes of psychotherapists towards their patients are more engaging than the more traditional neutral stance often assumed by psychotherapists. Adolescent-therapist agreement (i.e. co-endorsement of etiological beliefs) may significantly facilitate treatment engagement through promoting communication/openness, adolescent-therapist interaction, and adolescent's perceived usefulness of treatment [64].

Oetzel and Scherer (2003) arguably state that 'succeeding as a psycho-therapist with adolescents can be challenging' [27]. The authors state: 'establishing a strong therapeutic alliance with adolescents require that therapists express empathy and genuineness, utilize developmentally appropriate interventions, address the stigma, and increase choice in therapy' [27].

\section{Empathy, support and sincerity}

Many participants expressed their need to feel that their therapist will understand them and that he or she will be a source of support. Participants appreciated the fact that the therapist gave them thoughtful and effective advices. This is in consistency with prior literature [62]. They perceived their therapist as empathic, caring, open, and sincere.

Some participants said: 'the therapist understands me'. It is true that both therapist and adolescent should be minded to 'understand'. Indeed, reciprocal understanding between adolescent and therapist is of great importance. Understanding how adolescents perceive mental illness may be important for therapists to improve engagement [65]. If providers motivate adolescents to understand the value of treatment they may increase engagement of adolescents who will be less likely to terminate prematurely [66]. It is crucial to bear in mind that adolescents may undervalue or overestimate the importance of their psychological symptoms and may be ashamed of reporting them [27]. It is possible to identify different treatment engagement profiles [67]. Note, however, that treatment motivation should be distinguished from treatment engagement [68]. Motivational interviewing, used as a pre-treatment intervention, is a promising intervention to facilitate engagement in adolescent mental health settings [69].

Empathy, is necessary for developing a therapeutic alliance with adolescents, but is not sufficient. Adolescents appreciate therapists who are committed to them and their well-being.

Some participants said that they wanted the therapist to be precise and for them to feel comfortable with him. Sincerity, candor or "being real" with adolescents is a crucial therapy engagement facilitator. Candor implies telling adolescent clients the truth (tailored to the adolescents' developmental capacities). 
Cognitively immature adolescents require the therapist to use simple inquiries devoid of abstract terms, concrete examples, and guidance in how to establish therapeutic rapport [27].

\section{Respectforadolescents' values}

Some participants stated they were willing to interrupt treatment, if it was contradicting their values. Respect to the personal values of the adolescent is crucial both during the therapist's approach and for the adolescent's therapeutic goals. In relation to the above, it should be mentioned that each minor experiences the outside world in his own unique way, even though he lives in the same social-cultural context with other children [70].

All the aforementioned features of a therapeutic relationship are necessary to develop an effective shared decision making process that predicts effective treatment engagement of adolescents. Therapists should establish a climate that enables a thorough exchange with adolescents and their families, which allows for a flexible and respectful shared decision-making process [71, 72]. Furthermore, therapists should balance the views of parents and children [73], while making every effort to involve the adolescent's family in the decision-making process $[26,74,75]$.

It is significant that therapists should go beyond the provision of adequate, clear, concise and unbiased information to the patient [76]. Physicians have to empower and activate the adolescents to fully engage them in the process of (shared) making decisions with their own values intelligible from his or her own viewpoint, preferences and emotions [3,76]. Importantly, irrational decisions of adolescents which, however, are coherent with their 'internal rationality' may be regarded as internally reasonable decisions [77]. Shared decision-making (SDM) 'is increasingly being suggested as an integral part of mental health provision' [78], especially in the context of children and adolescents psychiatry [79]. However, while a subset of adolescents are decision making competent $[8,80]$, therapists may have challenges engaging adolescents with mental disorders in shared decision-making [78,81].

\section{Peers}

Acceptance by peers and improvement of social skills as an essential precondition for acceptance by the social-friendly environment seems to constitute a strong motive for seeking treatment. This is not surprising. Adolescents pursue their needs for independence by placing a considerable emphasis on attempting to shift from dependency on parents and family towards a greater "fit-in" with peers [27]. Many writers highlight the participation of adolescents in groups of peers as a necessary process of maturation, experimentation and finally discovery of the true self. [82-84].

Potential social stigma against mental illness and inability to deal alone with their difficulties pushes adolescents to conceal their receiving treatment from the social cycle, whereas simultaneously they seek help so as to integrate into it and to develop trusted relationships with peers. Mental disorder-related stigma attitudes of peer groups towards adolescents in psychotherapy may result in adolescents feeling scorned [27]. 
However, our research has shown that stable and tested friendly relationships could function encouragingly towards therapy and that there is distinction between "company" and "friends". In that regard, it should be highlighted that peers, even though they often are not part of medical conversation, actually may motivate a (mentally or not) ill adolescent to be more socially active, thus improving his or her decision making competence [13]. Indeed, in a peer context an observed adolescent may want to send a social signal to his or her peers [19]. The developmental processes that underlie the sensitivity of adolescents to peer influence are poorly understood [19]. At any rate, it should be highlighted that the influence of peers on outcomes in psychiatric mental health contexts remain poorly understood [9].

\section{Adolescents' aspirations and expectations}

Most participants did not correlate their goals for the future with their present treatment. However, for some, the realization of their aspirations and expectations constituted a facilitator of their treatment engagement. In that connection, it is important to note Almroth et al. (2018) state that 'interventions aimed at increasing aspirations and engagement in school may prevent mental health problems in adolescence' [85]. Furthermore, leaving home and university studies seem to be important inscriptions for the adolescents in the research and an element of autonomy and independence from family safety and protection. The selection of a foreign country for studies appears as an ideal choice which equals social recognition and financial independence and may reflect the general perception of young people about the value crises and financial poorness in their country (Greece) and so a foreign country appears ideal.

\section{Family}

In almost all of the cases adolescents were backed by family to a greater or lesser extent. Adolescents in the study acknowledge the important role family plays in their decisions. Tsiantis et al (1994)(86) state that when an adolescent comes for therapy, he has already received familial, friendly and social influences and this can make his attitude towards treatment positive [86].

It is very important that parents first note there is a problem and persuade the adolescent to seek treatment because, as participant.. said, "if the family doesn't accept the problem, then nor does the child" $[29,86]$. Although this was not the case for the participants in our study, adolescents often underestimate the importance of and need for treatment and are involuntarily referred by parents and other health care providers [87]. Furthermore, not only in mental health care but also in other health care contexts parents may facilitate adolescent's decision making competence more than physicians do, creating the context for adolescents' competent decision making [78]. Parents can be a barrier or facilitator of an adolescent's treatment decision [88]. American Academy of Pediatrics (2016) arguably state that there is not an absolute legal right of parents to make their own autonomous treatment decisions regarding their children [8]. There is parents' responsibility to preserve family relationships and support the best interest of their children. According to the model of constrained parental autonomy parents can "balance the "best interest" of the minor patient with his or her understanding of the family's best interests as long as the child's basic needs, medical and otherwise, are met' [8]. At any rate it is important to highlight the fact that parents (and physicians) do not always understand adolescent's best interest [72,89]. When parents 
perceive their children's mental health problem to be serious, they are more likely to seek mental health therapy for their children $[48,90]$. However, while the majority of families perceive the need for treatment, that perceived need may not be associated with treatment engagement [91].

Adolescents' and parents' needs and perceptions regarding need for and barriers to care may differ. It is important to align adolescents' and parents' needs throughout treatment [88]. Moreover, a collaborative relationship between the family and the health provider may increase engagement [33].

Additionally, it is important to bear in mind that the influences of parents and family on outcomes in psychiatric mental health contexts are poorly understood [9].

\section{Stigma}

The fear of mental health-related stigma emerged in the interview excerpts regarding the intervieweetherapist and interviewee-peers relationships. It is to be noted the role of the stigma that many adolescents associate with psychotherapy. In literature, stigma associated with mental illness is most commonly reported as potential (and fundamental) attitudinal barrier to seeking and engaging in mental health treatment among adolescents with mental disorders [48, 56, 60-63, 92, 93].

Unlike previous research [48], the participants in our study did not tell they had lived bad patient experiences in the hospital. Financial or infrastructural barriers that prevent access to care was not identified as affecting adolescents' treatment engagement or health-seeking behaviors for mental health care.

\section{Limitations}

We did not explore the perceptions of the participants' families. Furthermore, we did not explore how developmental factors may help or hinder therapeutic engagement. Moreover, as in our study we combined participants of a broad spectrum of diagnosis areas and some of the participants in our study could not be classified in mutually exclusive diagnosis areas, we did not conduct different data analysis for each diagnosis-related group.

\section{Implications}

For the most part, the findings enhance prior studies. However, we identified some nuances that can be used to inform the development of interventions that might contribute to enhancing facilitation techniques and reducing barriers to mental health care engagement among adolescents. For instance, by the subsiding of symptoms adolescents are most likely to terminate the treatment prematurely. To address it, therapists should highlight the long-term benefits of therapy. Moreover, therapists should examine the association between these long-term benefits and the achievement of adolescents' future goals as well as the prospect of achieving family's enhanced well-being because of the improvement of their mental health. Being psycho-therapist with adolescents is challenging and deserves to be supported. 


\section{Conclusions}

All the participants highlighted their experience with facilitators rather than barriers to their treatment engagement. Facilitators included: a) positive treatment outcomes and adolescent's perceived usefulness of treatment, b) a meaningful adolescent-therapist relationship, c) family's supportive role, d) symptoms and negative self-image, and e) developing social relationships and acceptance by peers. The participants equally highlighted the importance of getting rid of their symptoms and improving their socialization skills. Other facilitators included: achieving professional and personal goals in life, enhancing their independence and self-esteem / self-image, or even just confessing to another trustworthy person. Friends were reported as having a neutral or mild supportive role. The barriers included ineffective and unhelpful treatment, negative experiences with their therapist, relief from symptoms and mental health therapy-related stigma. Adolescents' interactions with their social ecology might be a facilitator or a barrier to their treatment engagement. Participants' most relevant symptoms were sleep disorders, mood disorders, depressive mood, lack of sexual interest, concentration difficulties, loss of energy.

For the most part, the findings of our study are in consistency with the findings of previous research on the topic of interest. It enhances the findings of prior studies. It is noteworthy that the (considered as) decision making competent participants in our study had a (mostly strong) positive attitude towards their treatment engagement. Furthermore, we identified some nuances which (to some extent) extend the findings of previous studies and might be used by therapists to enhance the adolescents' treatment engagement. For instance, bearing in their mind that by the subsiding of symptoms adolescents are most likely to terminate the treatment prematurely and highlighting the achievement of adolescents' future goals as well as the prospect of achieving family's enhanced well-being because of the improvement of adolescents' mental health may contribute to reducing the attrition rate. This implication for therapists can be used to guide them and lead them to assume more responsibility and initiative for enhancing treatment engagement in adolescents with mental disorders.

\section{Abbreviations}

DMC

Decision Making Competence / Capacity

SDM

Shared Decision Making

DMI

Decision Making Involvement

\section{Declarations}

\section{Authors' contributions}


ET and PV developed the study concept and design. All interviews were conducted by ET. ET and PV analyzed and interpreted the data. The other co-authors ( $A E$ and GA) were involved in data analysis discussions, monitored the process of this study and help responded to the reviewers' advises and comments. PV drafted the manuscript, and all authors provided critical revisions for important intellectual content. The study was supervised by PV. All authors read and approved the final manuscript. ET and PV (corresponding author) equally contributed to this paper.

\section{Acknowledgments}

The authors would like to thank the staff of the Department of Child \& Adolescent Psychiatry, Hippokration GeneralHospital of Thessaloniki (Greece), for their valuable cooperation for the recruitment of participants. They would also like to thank the participants of the present study and their families for their generous contribution.

\section{Competing interests}

The authors declare that they have no competing interests.

\section{Availability of data and materials}

The datasets used and analysed for this study are available from the corresponding author on reasonable request.

\section{Consent for publication}

Not applicable.

\section{Ethics approval and consent to participate}

This study and consent procedure was approved and monitored by the Research Ethics Review Board of the School of Medicine, Faculty of Health Sciences, Aristotle University of Thessaloniki, Greece (No: 9.302 / 12-07-2017). Before each interview, each participant and his or her parent(s) were given information on the study and informed that his or her participation was voluntary, while placing great weigh on the importance of maintaining confidentiality.

\section{Funding}

There are no sources of funding to be declared.

\section{Author information}

\section{Affiliations}

1. Department of Child \& Adolescent Psychiatry, Hippokration GeneralHospital of Thessaloniki, Konstantinoupoleos 49, Postal Code 54642 Thessaloniki, Greece. 
Tsamadou (Child \& Adolescent Psychiatrist, MSc in Bioethics)

2. Laboratory of Forensic Medicine \& Toxicology (Medical Law and Ethics), School of Medicine, Faculty of Health Sciences, Aristotle University, Campus, Postal Code 541 24, Thessaloniki, Greece.

Voultsos (Associate Professor)

3. Department of Child \& Adolescent Psychiatry, General Hospital of Volos, Polymeri 146, Postal Code 38222, Volos,

Emmanouilidis (Child \& Adolescent Psychiatrist)

4. Department of Child \& Adolescent Psychiatry, School of Medicine, Faculty of Health Sciences, Aristotle University, Campus, Postal Code 541 24, Thessaloniki, Greece.

Ampatzoglou, Professor Emeritus of Child \& Adolescent Psychiatry.

\section{Corresponding author}

Correspondence to Polychronis Voultsos.

\section{References}

1 Radoilska L. Autonomy and Mental Disorder Oxford: Oxford University Press, 2012, 328pp

2 Friedman M. Autonomy, Gender, Politics. Oxford: Oxford University Press, 2003.

3 Hermann H. et al. (2016). Hermann H, Trachsel M, Elger BS, Biller-Andorno N. Emotion and Value in the Evaluation of Medical Decision-Making Capacity: A Narrative Review of Arguments. Front Psychol. 2016 May 26;7:765.

4 Hartley CA, Somerville LH. The neuroscience of adolescent decision-making. Curr Opin Behav Sci. 2015;5:108-115.

5 Larcher V. Consent, competence, and confidentiality. BMJ. 2005;330(7487):353-356.

6 Miller VA. Involving Youth With a Chronic Illness in Decision-making: Highlighting the Role of Providers. Pediatrics. 2018;142(Suppl 3):S142-S148.

7 Santelli JS, Rosenfeld WD, DuRant RH, et al. Guidelines for adolescent health research: a position paper of the society for adolescent medicine. J Adolesc Health. 1995;17(5):270-276.

8 Katz AL, Webb SA, AAP COMMITTEE ON BIOETHICS. Informed Consent in Decision-Making in Pediatric Practice. Pediatrics. 2016;138(2):e20161485 
9 Roberson AJ, Kjervik DK. Adolescents' perceptions of their consent to psychiatric mental health treatment. Nurs Res Pract. 2012; 2012: 379756.

10 Sibley A, Pollard AJ, Fitzpatrick R, Sheehan M. Developing a new justification for assent. BMC Med Ethics. 2016;17:2.

11 den Hartogh, G. Do we need a threshold conception of competence?. Med Health Care and Philos 2016; 19: 71-83.

12 Dickens B, Cook RJ. Adolescents and Consent to Treatment. International Journal of Gynecology and Obstetrics, 2005; 89: 179-184. U Toronto Legal Studies Research Paper No. 14-05, Available from SSRN: https://ssrn.com/abstract=862384 (last access: 9 Sept 2020).

13 Schwartz Y, Williams TS, Roberts SD, Hellmann J, Zlotnik Shaul R. Adolescent decision-making in Canadian medical contexts: Integrating neuroscience and consent frameworks. Paediatr Child Health. 2018;23(6):374-376.

14 Hein IM, De Vries MC, Troost PW, Meynen G, Van Goudoever JB, Lindauer RJ. Informed consent instead of assent is appropriate in children from the age of twelve: Policy implications of new findings on children's competence to consent to clinical research. BMC Med Ethics. 2015b;16(1):76.

15 Parekh, S.A. (2006) 'Child consent and the law: an insight and discussion into the law relating to consent and competence', Child: Care, Health and Development, 33(1), pp. 78-82.

16 Schwartz Y, Williams TS, Roberts SD, Hellmann J, Zlotnik Shaul R. Adolescent decision-making in Canadian medical contexts: Integrating neuroscience and consent frameworks. Paediatr Child Health. 2018;23(6):374-376.

17 Grootens-Wiegers P, Hein IM, van den Broek JM, de Vries MC. Medical decision-making in children and adolescents: developmental and neuroscientific aspects. BMC Pediatr. 2017;17(1):120.

18 Ruggeri A, Gummerum M, Hanoch Y. Braving difficult choices alone: children's and adolescents' medical decision making. PLoS One. 2014;9(8):e103287.

19 Ciranka S, van den Bos W. Social Influence in Adolescent Decision-Making: A Formal Framework. Front Psychol. 2019;10:1915.

20 Tan JOA, Fegert JM. Capacity and Competence in Child and Adolescent Psychiatry. Health Care Analysis 2004; 12: 285-294.

21 Campbell AT. Consent, Competence, and Confidentiality Related to Psychiatric Conditions in Adolescent Medicine Practice. Adolescent Medicine Clinics 2006; 17(1):25-47. 
22 Tan JOA., Passerini GE, Stewart A. Consent and confidentiality in clinical work with young people', Clinical Child Psychology and Psychiatry 2007; 12(2): 191-210.

23 Didcock EA. Issues of consent and competency in children and young people. Paediatrics and Child Health 2007;17(11): 425-428.

24 Alderson, P. (2007) 'Competent children? Minors' consent to health care treatment and research', Social Science and Medicine, 65, pp. 2272-2283.

25 Larcher V, Hutchinson A. How should paediatricians assess Gillick competence? Archives of Disease in Childhood 2009; 95: 307-311.

26 Diekema DS. Adolescent Brain Development and Medical Decision-making. Pediatrics. 2020 Aug;146(Suppl 1):S18-S24.

27 Oetzel, K.B. and Scherer, D.G. Therapeutic Engagement With Adolescents in Psychotherapy. (2003). Psychotherapy Theory Research \& Practice 40(3):215-225

28 Gómez-Vírseda, C., de Maeseneer, Y. \& Gastmans, C. Relational autonomy in end-of-life care ethics: a contextualized approach to real-life complexities. BMC Med Ethics 2020; 21: 50.

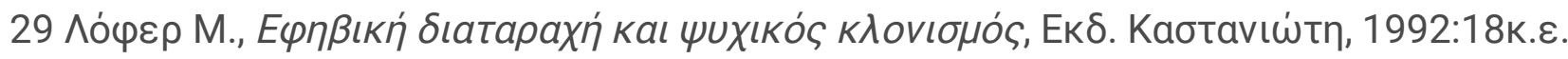

30 Merikangas, K. R., He, J., Burstein, M., Swanson, S. A., Avenevoli, S., Cui, L., et al. (2010b). Lifetime prevalence of mental disorders in U.S. adolescents: Results from the National Comorbidity Survey Replication-Adolescent Supplement (NCS-A). Journal of the American Academy of Child and Adolescent Psychiatry, 49(10), 980-989.

31 Dean S, Britt E, Bell E, Stanley J, Collings S. Motivational interviewing to enhance adolescent mental health treatment engagement: a randomized clinical trial. Psychol Med. 2016 Jul;46(9):1961-9.

32 Thompson SJ, Bender K, Lantry J, Flynn PM. Treatment Engagement: Building Therapeutic Alliance in Home-Based Treatment with Adolescents and their Families. Contemp Fam Ther. 2007 Jun;29(1-2):39-55.

33 Warnick EM, Bearss K, Weersing VR, Scahill L, Woolston J. Shifting the treatment model: impact on engagement in outpatient therapy. Adm Policy Ment Health. 2014 Jan;41(1):93-103.

34 Tan JOA., Passerini GE, Stewart A. Consent and confidentiality in clinical work with young people', Clinical Child Psychology and Psychiatry 2007; 12(2): 191-210.

35 Woolger C. Wechsler Intelligence Scale for Children-Third Edition (WISC-III). In: Dorfman W.I., Hersen M. (eds) Understanding Psychological Assessment. Perspectives on Individual Differences. Springer, Boston, MA. 2001. https://doi.org/10.1007/978-1-4615-1185-4_11C. 
36 Beck, A.T., Steer, R.A., \& Brown, G.K. (1996). Manual for the Beck Depression Inventory-II. San Antonio, TX: Psychological Corporation.

40 Zhang Y. and Wildemuth B.M. "Qualitative Analysis of Content," In: B. M. Wildemuth, Ed., Applications of Social Research Methods to Questions in Information and Library Science, Libraries Unlimited, 2009. pp. 1-12.

41 Graneheim UH, Lundman B. Qualitative content analysis in nursing research: concepts, procedures and measures to achieve trustworthiness. Nurse Educ Today. 2004 Feb;24(2):105-12.

42 Gibbs G. The Sage qualitative research kit. Analyzing qualitative data. Sage Publications Ltd. 2007.

43 Lincoln YS, Guba EG. Naturalistic Inquiry. Newbury Park, CA: Sage Publications. 1985.

44 Guba EG, Lincoln YS. Competing paradigms in qualitative research. In N. K. Denzin and Y. S. Lincoln (Eds.), Handbook of qualitative research 1994, p. 105-117.

45 Elo S. Kynga“s. The qualitative content analysis process. Journal of Advanced Nursing 2008; 62(1): 107-115.

46 Burnard P, Gill P, Stewart K, Treasure E, Chadwick B. Analysing and presenting qualitative data. Br Dent J. 2008 Apr 26;204(8):429-32.

47 Patton MQ. Two Decades of Developments in Qualitative Inquiry: A Personal, Experiential Perspective. Qualitative Social Work 2002; 1(3): 261-283.

48 Stafford AM, Draucker CB. Barriers to and Facilitators of Mental Health Treatment Engagement Among Latina Adolescents. Community Ment Health J. 2020 May;56(4):662-669.

49 Schachter D, Kleinman I, Harvey W. Informed consent and adolescents. Can J Psychiatry. 2005;50(9):534-540.

50 Rushforth $\mathrm{H}$. Practitioner review: communicating with hospitalised children: review and application of research pertaining to children's understanding of health and illness. Journal of child psychology and psychiatry, and allied disciplines 1999; 40(5): 683-91.

51 Barrett MS, Chua WJ, Crits-Christoph P, Gibbons MB, Casiano D, Thompson D. Early withdrawal from mental health treatment: implications for psychotherapy practice. Psychotherapy (Chic). 2008 Jun 1;45(2):247-267.

52 Surtees AD, Apperly IA. Egocentrism and automatic perspective taking in children and adults. Child Dev. 2012 Mar-Apr;83(2):452-60.

53 Wickman ME, Koniak-Griffin D. Invincibility fable: tool development to measure invincibility. J Pediatr Nurs. 2013 Nov-Dec; 28 (6):575-84. 
54 Hardoff D. Legal and ethical issues in adolescents' health care. Georgian Med News. 2012 Sep; (210):18-23. English, Georgian.

55 Cabassa LJ, Hansen MC, Palinkas LA, Ell K. Azucar y nervios: Explanatory models and treatment experiences of Hispanics with diabetes and depression. Social Science \& Medicine 2008; 66(12): 24132424.

56 Green BL, Watson MR, Kaltman SI, Serrano A, Talisman N, Kirkpatrick L, et al. Knowledge and preferences regarding antidepressant medication among depressed Latino patients in primary care. The Journal of Nervous and Mental Disease 2017; 205(12): 952-959.

59 Uebelacker LA, Marootian BA, Pirraglia PA, Primack J, Tigue PM, Haggarty R. et al. Barriers and facilitators of treatment for depression in a Latino community: A focus group study. Community Mental Health Journal 2012; 48(1): 114-126.

70 Greene, S., \& Hill, M. (2005). Researching children's experience: Methods and methodological issues. In S. Greene \& D. Hogan (Eds.), Researching children's experience. Approaches and methods (pp.1 - 21). London: Sage.

71 Michaud PA. Assessing adolescent capacity for decision making in clinical care: the practical application of bioethics and human rights principles. In: Cherry A, Baltag V, Dillon M, editors. International handbook on adolescent health and development. Switzerland: Springer; 2017. p.359-372.

72 Alderson P, Sutcliffe K, Curtis K. Children's competence to consent to medical treatment. Hastings Cent Rep. 2006; 36(6):25-34.

73 Cox A., Brannigan C., Martyn H., Townend M. The factors that influence decision making by 8-12 year olds in Child and Adolescent Mental Health Services (CAMHS): a systematic review. Research, Policy and Planning: The Journal of the Social Services Research Group 2016; 31(3): 195-209.

74 Ruggeri A, Gummerum M, Hanoch Y. Braving difficult choices alone: children's and adolescents' medical decision making. PLoS One. 2014;9(8):e103287.

75 Larcher V. Consent, competence, and confidentiality. BMJ. 2005;330(7487):353-356.

76 Ubel PA, Scherr KA, Fagerlin A. Empowerment Failure: How Shortcomings in Physician Communication Unwittingly Undermine Patient Autonomy. Am J Bioeth. 2017;17(11):31-39.

77 Charland, LC. In defence of emotion. Canadian Journal of Philosophy 2001; 31 (1): 133-154.

78 Hayes, D., Edbrooke-Childs, J., Town, R. et al. Barriers and facilitators to shared decision making in child and youth mental health: clinician perspectives using the Theoretical Domains Framework. Eur Child Adolesc Psychiatry 2019; 28: 655-666. 
79 Hayes D, Fleming I, Wolpert M. Developing safe care in mental health for children and young people: drawing on UK experience for solutions to an under-recognised problem. Curr Treat Options Pediatr 2015; 1:309-319.

80 Parsapoor A, Parsapoor MB, Rezaei N, Asghari F. Autonomy of children and adolescents in consent to treatment: ethical, jurisprudential and legal considerations. Iran J Pediatr. 2014 Jun;24(3):241-8.

81 Yoo A, Kim M, Ross MM, Vaughn-Lee A, Butler B, dosReis S. Engaging Caregivers in the Treatment of Youth with Complex Developmental and Mental Health Needs. J Behav Health Serv Res. 2018 Jul;45(3):440-453.

82 Braconnier A., Marcelli D. [The thousand faces of adolescence]. Athens: Kastaniotis, 2002, pp: 30 et seq. [in Greek]

83 Braams B. R., Davidow J. Y., Somerville L. H. (2019). Developmental patterns of change in the influence of safe and risky peer choices on risky decision-making. Dev. Sci. 22:e12717.

$10.1111 /$ desc. 12717

84 Steinberg L. (2008). A social neuroscience perspective on adolescent risk-taking. Dev. Rev. 28 78-106. 10.1016/j.dr.2007.08.002

85 Almroth MC, László KD, Kosidou K, Galanti MR. Association between adolescents' academic aspirations and expectations and mental health: a one-year follow-up study. Eur J Public Health. 2018 Jun 1;28(3):504-509.

86 Tsiantis et al., [Adolescence, a transitional .stage in a changing world.] Athens: Kastaniotis, 1994, pp: 94 et seq [in Greek].

87 Digiuseppe R, Linscott J, Jilton R. Developing the therapeutic alliance in child-adolescent psychotherapy. Applied and Preventive Psychology. 1996;5(2):85-100.

88 Schnyder N, Lawrence D, Panczak R, Sawyer MG, Whiteford HA, Burgess PM, Harris MG. Perceived need and barriers to adolescent mental health care: agreement between adolescents and their parents. Epidemiol Psychiatr Sci. 2019 Sep 20;29:e60.

89 Dreger A. One Of Us: Conjoined Twins and The Future of Normal. Cambridge MA: Cambridge University Press, 2004.

90 Reardon, T., Harvey, K., Baranowska, M., O’Brien, D., Smith, L., Creswell, C. What do parents perceive are the barriers and facilitators to accessing psychological treatment for mental health problems in children and adolescents? A systematic review of qualitative and quantitative studies. European Child \& Adolescent Psychiatry 2017; 26(6): 623-647. 
91 Fisher JH, Lichvar E, Hogue A, Dauber S. Perceived Need for Treatment and Engagement in Mental Health Services Among Community-Referred Racial/Ethnic Minority Adolescents. Adm Policy Ment Health. 2018 Sep;45(5):751-764.

92 Gearing RE, Brewer KB, Schwalbe CS, Mackenzie MJ, Ibrahim RW. Stigma and adolescents with psychosis in the Middle East: implications for engaging in mental health treatment. J Nerv Ment Dis. 2013 Jan;201(1):68-71.

93 Gearing RE, MacKenzie MJ, Ibrahim RW, Brewer KB, Batayneh JS, Schwalbe CS. Stigma and mental health treatment of adolescents with depression in jordan. Community Ment Health J. 2015 Jan;51(1):111-7.

\section{Supplementary Files}

This is a list of supplementary files associated with this preprint. Click to download.

- ADOLESCENTSADDITIONALFILE.doc 